\title{
Reliability based robust design optimization of steel structures
}

\author{
Nikos D. Lagaros ${ }^{\mathrm{a}}$, Vagelis Plevris and Manolis Papadrakakis \\ Institute of Structural Analysis \& Seismic Research, National Technical University of Athens, 9, Iroon Polytechniou Str., \\ Zografou Campus, GR-15780 Athens, Greece
}

Received 21 August 2007; accepted 15 September 2007

\begin{abstract}
In this work the uncertainty of a structural system is taken into account in the framework of a structural Reliability based Robust Design Optimization (RRDO) formulation where probabilistic constraints are incorporated into the robust design optimization formulation. A robust design optimization problem is formulated as a multi-criteria optimization problem. The Pareto front representing the solution of the RRDO problem is composed by designs with a state of robustness, since their performance is the least sensitive to the variability of the uncertain variables. The cross section dimensions together with other structural parameters, such as the modulus of elasticity, the yield stress and the applied loading, are considered as random variables. For the solution of the RRDO problem, the non-dominant Cascade Evolutionary Algorithm is employed combined with a weighted Tchebycheff metric.
\end{abstract}

Key words: Probabilistic constraints; robust design optimization; cascade evolutionary algorithms; Monte Carlo simulation

\section{Introduction}

Improving the performance of a structural system in terms of constructional cost and structural response is the main objective during its development. Improvements can be achieved either by simply using design rules based on the experience or on an automated way using optimization methods. Strictly speaking, optimal means that no better solution exists under certain constraints. Considering the complexity of a structural optimization problem it is obvious that finding the global optimum solution is a very difficult task. In the real world, given the uncertainty or scatter of the structural parameters, the significance of such an optimum solution, where the uncertainty has not been taken into account, would be limited. Although in a computing environment nearly perfect structural models can be simulated, real world structures always have imperfections or deviations from the nominal state defined by the design codes. An optimum solution obtained computationally is never materialized in an absolute way and as a result a near optimal solution is always implemented in practice. A deterministic based formulation of a structural optimization problem ignores scatter of any kind of the structural parameters. It is possible to achieve a feasible optimum according to the deterministic formulation, yet once this solution is implemented in a real physical system, its optimal performance may vanish due to of the parameters scatter which is unavoidable. Consequently,

\footnotetext{
a Corresponding author:

\{nlagaros, vplevris, mpapadra\}@central.ntua.gr
}

the performance of the "implemented" design may be far worse than expected. In order to account for the randomness of some parameters that affect the response of the structure, a different formulation of the optimization problem has to be used based on stochastic analysis.

In recent years, probabilistic based formulations of the optimization problem have been developed to account for uncertainty and randomness through stochastic simulation and probabilistic analysis. The development, over the last two decades [1], on the stochastic analysis methods has stimulated the interest for probabilistic structural optimization problems. There are two distinguished design formulations to account for the probabilistic systems response: Robust Design Optimization (RDO) [2-4] and Reliability-Based Design Optimization (RBDO) [5-8]. RDO formulations primarily seek to minimize the influence of stochastic variations on the mean design. On the other hand, the main goal of RBDO formulations is to design for safety with respect to extreme events. In this work a Reliability based Robust Design Optimization (RRDO) formulation is examined, where probabilistic constraints are incorporated into the RDO formulation.

The main goal of this work is to account for the influence of probabilistic constraints in the framework of a structural RDO problem, by comparing the RRDO formulation with the RDO one. The objective functions considered for both formulations are the weight and the variance of the response of the structure represented by a characteristic node displacement. In the case of the RDO 
formulation each design is checked whether it satisfies the provisions of the European design codes for steel structures [9]. On the other hand, additional probabilistic constraints are taken into account in the case of the RRDO formulation, where the code provisions are checked if they are satisfied with a prescribed probability of violation. The uncertainty of loads, material properties, and cross section dimensions is taken into consideration using the Monte Carlo Simulation (MCS) method combined with the Latin hypercube sampling. The solution of the multi-criteria optimization problem is performed with the non-dominant Cascade Evolutionary Algorithm combined with a weighted Tchebycheff metric.

\section{Deterministic based structural optimization}

\subsection{Single objective optimization problem}

In a single objective Deterministic Based sizing Optimization (DBO) problem the aim is often to minimize the weight of the structure subject to certain deterministic behavioural constraints usually on stresses and displacements. A discrete DBO problem can be formulated in the following form

$$
\begin{aligned}
\min & f(\mathbf{s}) \\
\text { subjectto } & g_{j}(\mathbf{s}) \leqslant 0 \quad j=1, \ldots, k \\
& s_{i} \in R^{d}, \quad i=1, \ldots, n
\end{aligned}
$$

where $f(\mathbf{s})$ is the objective function, $\mathbf{s}$ is a vector of the design variables, which can take values only from a discrete given set $R^{d}$, while $g_{j}(\mathbf{s})$ are the deterministic constraints. Most frequently the deterministic constraints refer to the member stresses and nodal displacements or the interstorey drifts in building structures.

\subsection{Multi-objective optimization problem}

In practical applications of sizing optimization problems, the weight itself rarely gives a representative measure of the performance of the structure. In fact, several conflicting and usually incommensurable criteria usually exist in real-life design problems that have to be dealt with simultaneously. This situation forces the designer to look for a good compromise among the conflicting requirements. Problems of this kind are defined as multi-objective optimization problems. The consideration of such problems in their present form originated towards the end of the 19th century when Pareto [10] presented the optimality concept in economic problems with several conflicting criteria.

Criteria and conflict in multi-objective structural optimization

An engineer looking for the optimum design of a structure is faced with the question of selecting the most suitable criteria for measuring the economy, strength, serviceability or any other factor that affects the performance of the structure. Any quantity that has a direct influence on the structural performance can be considered as a criterion. On the other hand, quantities that must satisfy imposed requirements are not considered as criteria but they can be treated as constraints.

One important property in the multi-criteria formulation is the conflict that may exist among the criteria. Only those quantities that are competing with each other should be treated as independent criteria whereas the others can be combined into a single criterion representing the whole group. Two functions $f_{i}$ and $f_{j}$ are called locally collinear with no conflict at point $\mathbf{s}$ if there is $c>0$ such that $\nabla f_{i}(\mathbf{s})=c \nabla f_{j}(\mathbf{s})$. Otherwise, the functions are called locally conflicting at point $\mathbf{s}$. According to this definition any two criteria are locally conflicting at a point of the design space if their maximum improvement is achieved at different directions. On the other hand, two functions $f_{i}$ and $f_{j}$ are called globally conflicting in the feasible region $\mathrm{F}$ of the design space when the two optimization problems $\min \left\{f_{i}(\mathbf{s}), \mathbf{s} \in F\right\}$ and $\min \left\{f_{j}(\mathbf{s})\right.$, $\mathbf{s} \in F\}$ have different optimal solutions.

\section{Formulation of a multi-objective optimization problem}

The selection of the design variables, criteria and constraints represents undoubtedly the most important part in the formulation of an optimization problem. In general, the mathematical formulation of a multi-objective problem with $n$ design variables, $m$ objective functions and $k$ constraint functions and can be defined as follows

$$
\begin{aligned}
\min _{\mathbf{s} \in \mathrm{F}} & {\left[f_{1}(\mathbf{s}), f_{2}(s), \ldots, f_{m}(s)\right]^{T} } \\
\text { subject to } & g_{j}(\mathbf{s}) \leqslant 0 \quad j=1, \ldots, k \\
& s_{i} \in R^{d}, \quad i=1, \ldots, n
\end{aligned}
$$

where $\mathbf{s}$ is a design variable vector and $\mathrm{F}$ is the feasible set in the design space $R^{n}$ which is defined as the set of design variables that satisfy the constraint functions $g(\mathbf{s})$

$$
F=\left\{\mathbf{s} \in R^{n} \mid g_{j}(\mathbf{s}) \leqslant 0 \quad j=1, \ldots, k\right\}
$$

If the $m$ objective functions are globally conflicting, there exists no unique point which represents the optimum for all $m$ criteria. Thus the common optimality condition used in single-objective optimization must be replaced by a new concept, the so called Pareto optimum. A design vector $\mathbf{s} * \in \mathrm{F}$ is called Pareto optimum for the problem of equation (2) if and only if there is no other design vector $\mathrm{s} \in \mathrm{F}$ such that

$$
\begin{array}{ll}
f_{i}(\mathbf{s}) \leqslant f_{i}\left(\mathbf{s}^{*}\right) & \text { for } i=1, \ldots, m \\
\text { with } \quad f_{i}(\mathbf{s})<f_{i}\left(\mathbf{s}^{*}\right) & \text { for at least one objective } i
\end{array}
$$

The geometric locus of the Pareto optimum solutions is called Pareto front curve and represents the solution of the optimization problem with multiple objectives. A typical Pareto front curve is depicted in Figure 1 for two conflicting objective functions $f(x)$ and $g(x)$ to be minimized. 


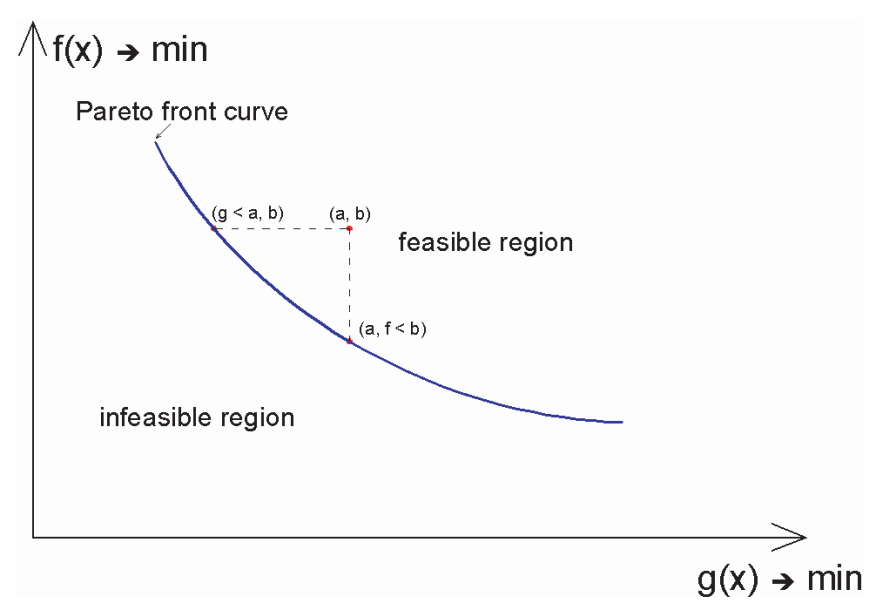

Fig. 1. Typical Pareto front curve.

\section{Solving the multi-objective optimization problem}

A number of techniques have been developed to deal with the multi-objective optimization problem $[11,12]$. The algorithm employed in this work belongs to the hybrid methods, where an evolutionary algorithm is combined with a scalarizing function. In general, when scalarizing functions are used, local Pareto optimal solutions are obtained. Global Pareto optimality can be guaranteed only when the objective functions and the feasible region are both convex or quasi-convex and convex, respectively. For non-convex cases, such as the majority of structural multiobjective optimization problems, a global single objective optimizer is required.

\section{Single objective optimizer}

The optimization methods for single objective problems can be classified in two general categories: deterministic and probabilistic. Mathematical Programming (MP) methods, such as Sequential Quadratic Programming (SQP) belong to the first category. The main advantage of such optimizers is that they capture the right path to the nearest optimum quickly, but they cannot guarantee that the global optimum has been achieved. Consequently, in real world structural optimization applications, MP algorithms encounter great difficulties in dealing with multiple local minima, large and non-convex search spaces and several (possibly conflicting) constraints that have to be satisfied [13].

On the other hand, probabilistic optimization techniques, such as Evolutionary Algorithms (EA), are robust and present a better global behaviour than the MP methods when dealing with complex optimization problems $[14,15]$. EA are not vulnerable to being trapped in local optima and therefore can be considered as reliable in approaching the global optimum for non-convex constrained optimization problems. Due to their random search, EA proceed toward the optimal solution with slower rate than gradient-based optimizers and most times need a greater number of analysis steps. However, these analyses are less time consuming than the corresponding computations in MP algorithms, since EA do not require gradient calculations. Thus, an EA-based optimization procedure is implemented in the present work for the solution of the single objective optimization part.

Augmented weighted Tchebycheff problem

The weighted Tchebycheff metric that was used by the authors in a previous work [16] is stated as follows

$$
\min _{s \in F} \max _{i=1, \ldots, m}\left[w_{i} \frac{\left|f_{i}(\mathbf{s})-z_{i}^{*}\right|}{f_{i}(\mathbf{s})}+\rho \sum_{i=1}^{m} \frac{\left|f_{i}(\mathbf{s})-z_{i}^{*}\right|}{f_{i}(\mathbf{s})}\right]
$$

where $\rho$ is a sufficiently small positive scalar (in this work $\rho=0.1$ ) while $z_{i}^{*}$ is the utopian objective function value. The weight parameters $w_{i}$ are random numbers, uniformly distributed between 0 and 1 . These weight parameters have to fulfil the requirement

$$
\sum_{i=1}^{m} w_{i}=1
$$

and if not, they are updated according to the following expression:

$$
w_{i}= \begin{cases}w_{i}+\frac{1-\sum_{i}^{m} w_{i}}{\sum_{i}^{m} w_{i}} w_{i}, & \text { if } \sum_{i}^{m} w_{i} \neq 1 \\ w_{i}, & \text { if } \sum_{i}^{m} w_{i}=1\end{cases}
$$

\section{Cascade optimization}

It is generally accepted that there is still no unique optimization algorithm capable of handling all existing optimization problems effectively. Cascade optimization has been proposed as a remedy to this observation $[15,17,18]$. According to this optimization strategy, a multi-stage procedure is applied, in which various optimizers are implemented successively. In the first stage of the cascade procedure the initial optimizer starts from a user-specified design known as 'cold-start'. The intermediate optimal solutions, called 'hot-starts', are used to initiate the next cascade stages. Each optimization stage of the cascade procedure starts from the (possibly perturbed) optimum design achieved in the previous stage. Thus, each cascade stage initiates from a hot-start and produces a new hotstart for the next stage, coupling the autonomous computations of successive optimization stages.

The optimization algorithm implemented in each stage of the cascade process (called cascade step) may or may not be the same. Cascade optimization has been implemented using different deterministic optimizers [17], as well as using both deterministic and probabilistic optimizers $[15,18]$. The main advantage in combining different optimizers in a successive manner has to do with maximizing the exploitation of the advantages offered by various optimization algorithms and alleviating the influence of the corresponding disadvantages on the optimum design achieved. The selection of the optimizers to be included in a cascade procedure, their exact sequence and 
the number of cascade stages performed can be determined via a trial-and-error process.

Non-dominant CEA multi-objective search using the Tchebycheff metric

In the present work the idea of Cascade Evolutionary Algorithms (CEA) is implemented for solving multiobjective structural optimization problems. The resulting cascade evolutionary procedure consists of a number of optimization stages, each of which employs the same EA optimizer. In order to differentiate the search paths followed by the same optimization algorithm during the cascade stages, the initial conditions of individual optimization runs are suitably controlled by using at each stage: (a) a different initial design (each stage initiates from the solution of the previous stage) and (b) a different seed for the random number generator of the EA process.

The multi-objective optimization procedure used in this study is based on the combination of the CEA procedure and the Tchebycheff metric employing the nondominant search. The multi-objective optimization procedure initiates with a set of parent vectors and a set of weighting coefficients for each independent run, representing a single objective optimization problem of the series. The independent runs can be implemented in parallel and in every global generation non-dominant search is applied. A global generation is defined as the state where a local generation (a generation in one of the independent runs) is completed in every independent run. The non-dominant search can be applied either in every global generation or locally for a number of local generations before it is applied globally. In this work the first option is used where each independent run corresponds to a CEA procedure using the Tchebycheff metric. According to this procedure in every global generation a local Pareto front is produced which evolves towards the global one. The basic idea of the procedure is to create an evolutionary process of the Pareto front curve.

The optimization algorithm used in this study is denoted as: non-dominant $C E A T m(\mu+\lambda)_{\text {nrun,csteps }}$ where $\mu, \lambda$ are the number of the parent and offspring vectors respectively, nrun is the number of independent CEA procedures and csteps is the number of cascade steps employed. A detailed description of the CEATm method can be found in a previous work of the authors [16]. The basic steps inside an independent run of the multi-objective algorithm, as adopted in this study, are the following:

\section{Independent run $i, i=1, \ldots$, nrun}

Generate the parameters $w_{i, j} j=1, \ldots, m$ of the Tchebycheff metric. Check if the requirement of equation (6) is fulfilled, if not change the weight parameters using equation (7).

\section{CEATm loop}

\section{Initial generation:}

1a. Generate $\mathbf{s}_{k}(k=1, \ldots, \mu)$ vectors

1b. Analysis step

1c. Evaluation of the Tchebycheff metric, equation (5)

1d. Constraint check: if satisfied $k=k+1$ else $k=k$. Go to step 1 a

2. Global non-dominant search: Check If global generation is accomplished. If yes, then non-dominant search is performed, else wait until global generation is accomplished.

3. New generation:

3a. Generate $\mathbf{s}_{\ell}(\ell=1, \ldots, \lambda)$ vectors

3b. Analysis step

3c. Evaluation of the Tchebycheff metric, equation (5)

3d. Constraint check: if satisfied $\ell=\ell+1$ else $\ell=\ell$. Go to step $3 a$

4. Selection step: selection of the next generation parents according to $(\mu+\lambda)$ or $(\mu, \lambda)$ scheme

5. Global non-dominant search: Check If global generation is accomplished. If yes, then non-dominant search is performed, else wait until global generation is accomplished.

6. Convergence check: If satisfied stop, else go to step 3 End of CEATm loop

\section{End of Independent run i}

\section{Reliability based robust design optimization}

In a reliability based robust design structural sizing optimization problem an additional objective function is considered which is related to the influence of the random nature of some structural parameters on the performance of the structure. In the present study the aim is to minimize both the weight and the variance of the response of the structure. In a RRDO problem the constraint functions are also varied due to variations of the random structural parameters. The mathematical formulation of the RRDO problem implemented in this study is as follows

$$
\begin{aligned}
\min & \Phi(\mathbf{s}, \mathbf{x}) \\
\text { subject to } & g_{j}(\mathbf{s}) \leqslant 0 \quad j=1, \ldots, k \\
& p_{v, j}(\mathbf{s}, \mathbf{x}) \leqslant p_{\text {all }} \quad j=1, \ldots, k \\
& s_{i} \in R^{d}, \quad i=1, \ldots, n
\end{aligned}
$$

where $\Phi(\mathbf{s})$ is the multi-objective function which is defined by equation (5), $\mathrm{s}$ is the vector of the design variables, which can take values only from the given discrete set $R^{d}, g_{j}(\mathbf{s})$ are the deterministic constraints while $p_{v, j}$ is the probability of violation of the $j$-th constraint bounded by an upper allowable probability equal to $p_{\text {all }}$, while $\mathbf{x}$ is the vector of the random variables. Probabilistic constraints define the feasible region of the design space by restricting the probability that a deterministic constraint is violated within the allowable probability of violation. The probabilistic constraints that are employed in this study enforce the condition that the probabilities of violation of the structure are smaller than a certain value.

Figure 2 depicts the difference between the DBO and the RDO optimum for a single variable problem. In the 


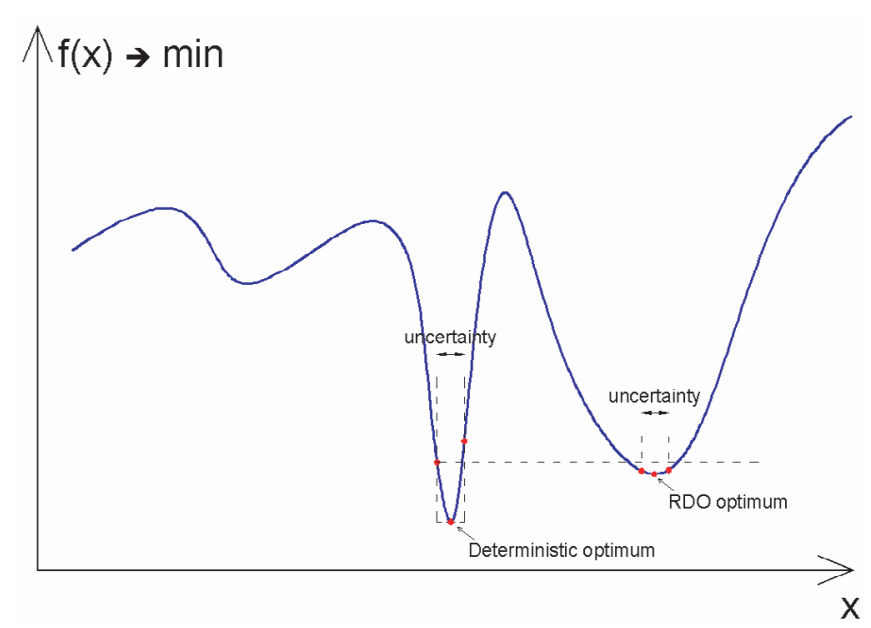

Fig. 2. The concept of robust design optimization.

case of DBO formulation the minimization of the objective function $f(x)$ is the only criterion, on the other hand according to the RDO formulation the minimization of the objective function $f(x)$ in conjunction with its variance is considered. It can be seen that the DBO formulation leads to a design having the minimum objective function value ignoring its variance while the RDO formulation leads to a compromise optimum solution corresponding a worse objective function value compared to the one of the DBO solution, but it is significantly better with respect to the variance.

In this study three types of constraints are imposed: (i) stress, (ii) compressive force (for buckling) and (iii) displacement constraints. The stress constraint can be written as follows

$$
\begin{aligned}
\sigma_{\max } & \leqslant \sigma_{a} \\
\sigma_{a} & =\frac{\sigma_{y}}{1.10}
\end{aligned}
$$

where $\sigma_{\max }$ is the maximum axial stress in each element group for all loading cases, $\sigma_{a}$ is the allowable axial stress according to Eurocode $3[9]$ and $\sigma_{y}$ is the yield stress. For the members under compression an additional constraint is used

$$
\begin{aligned}
\left|P_{c, \text { max }}\right| & \leqslant P_{c c} \\
P_{c c} & =\frac{P_{e}}{1.05} \\
P_{e} & =\frac{\pi^{2} E I}{L_{e f f}^{2}}
\end{aligned}
$$

where $P_{c, \text { max }}$ is the maximum axial compressive force for all loading cases, $P_{e}$ is the critical Euler buckling force in compression, taken as the first buckling mode of a pinconnected member, and $L_{\text {eff }}$ is the effective length. The effective length is taken equal to the actual length of each element. Similarly, the displacement constraints can be written as

$$
|d| \leqslant d_{a}
$$

where $d_{a}$ is the allowable value of the displacement at a certain node or the maximum nodal displacement.

\section{Monte Carlo simulation}

In stochastic analysis of structures the MCS method is particularly applicable when an analytical solution is not attainable. This is mainly the case in problems of complex nature with a large number of random variables (random structural parameters), where all other stochastic analysis methods are not applicable. Despite the fact that the mathematical formulation of the MCS is simple and the method has the capability of handling practically every possible case regardless of its complexity, this approach has not received an overwhelming acceptance due to the excessive computational effort that it requires. Despite the improvements achieved on the efficiency of the computational methods for treating reliability analysis problems, they still require disproportional computational effort for practical reliability problems. This is the reason why relatively few successful numerical investigations are known in the field. The Latin Hypercube Sampling (LHS) method was introduced by MacKay et al. [19] in an effort to reduce the required computational cost of purely random sampling methodologies. LHS is generally recognized as one of the most efficient size reduction techniques. In the current study the MCS with Latin hypercube samples has been employed for the calculation of the probability of violation of the behavioural constraints and the variance of the response of the structure which are required in the framework of an RDO problem.

In structural stochastic analysis problems, where the probability of violation of some behavioural constraints is to be calculated, MCS can be stated as follows: expressing the limit state function as $G(\mathbf{x})<0$, where $\mathbf{x}=\left[x_{1}, x_{2}\right.$, $\left.\ldots, x_{M}\right]^{\mathrm{T}}$ is a vector of the random structural parameters, the probability of violation of the behavioural constraints can be written as

$$
p_{\text {viol }}=\int_{G(x) \geqslant 0} f_{x}(\mathbf{x}) d \mathbf{x}
$$

where $f_{x}(\mathbf{x})$ denotes the joint probability of violation for all random structural parameters. Since MCS is based on the theory of large numbers $\left(N_{\infty}\right)$ an unbiased estimator of the probability of violation is given by

$$
p_{v i o l}=\frac{1}{N_{\infty}} \sum_{j=1}^{N_{\infty}} I\left(\mathbf{x}_{j}\right)
$$

where $\mathbf{x}_{j}$ is the $j$-th vector of the random structural parameters, and $I\left(\mathbf{x}_{j}\right)$ is an indicator for successful and unsuccessful simulations defined as

$$
I\left(x_{j}\right)=\left\{\begin{array}{lll}
1 & \text { if } & G\left(\mathbf{x}_{j}\right) \geqslant 0 \\
0 & \text { if } & G\left(\mathbf{x}_{j}\right)<0
\end{array}\right.
$$

In order to estimate $p_{\text {viol }}$ an adequate number of $N$ independent random samples is produced using a specific, uniform probability density function of the vector $\mathbf{x}_{j}$. The value of the violation function is computed for each random sample $\mathbf{x}_{j}$ and the Monte Carlo estimation of $p_{v i o l}$ 


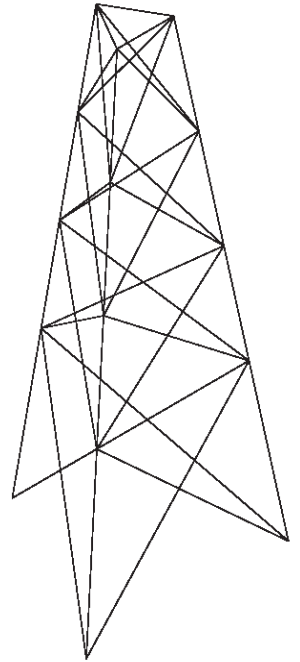

(a)

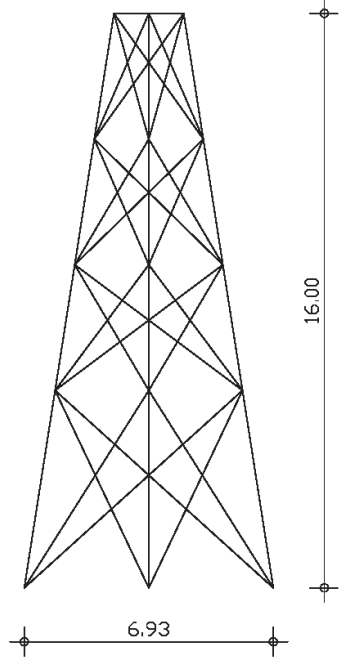

(b)

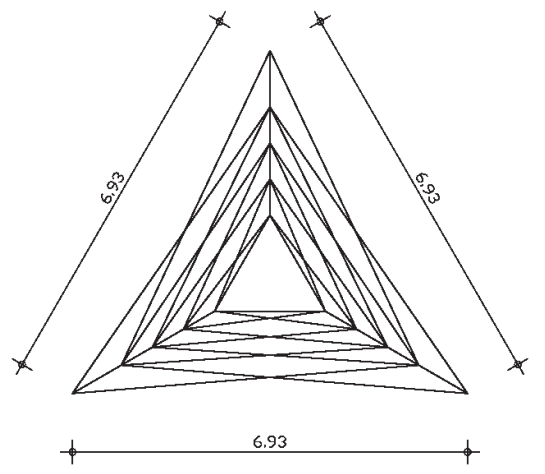

(c)

Fig. 3. 39-bar truss: (a) 3D view, (b) Side view, (c) Top view.

Table 1. 39-bar truss example: characteristics of the random variables.

\begin{tabular}{ccccc}
\hline \multicolumn{2}{c}{ Random variable } & $\begin{array}{c}\text { Probability } \\
\text { Density } \\
\text { Function }\end{array}$ & $\begin{array}{c}\text { Mean value } \\
\mu\end{array}$ & CoV \\
\hline $\mathbf{E ~}\left(\mathbf{k N} / \mathbf{m}^{2}\right)$ & Young's Modulus & Normal & $2.10 \mathrm{E}+08$ & $7.14 \%$ \\
$\sigma_{y}\left(\mathbf{k N} / \mathbf{m}^{2}\right)$ & Allowable stress & Normal & $3.55 \mathrm{E}+05$ & $10.00 \%$ \\
$\mathbf{F ~}(\mathbf{k N})$ & Horizontal loading & Normal & 8 & $37.50 \%$ \\
$\mathbf{D}$ & CHS Diameter & Normal & $\mathrm{d}_{i} *$ & $2 \%$ \\
$\mathbf{t}$ & CHS Thickness & Normal & $\mathrm{t}_{i} *$ & $2 \%$ \\
\hline
\end{tabular}

* Taken from the Circular Hollow Section (CHS) table of the Eurocode, for every design

is given in terms of sample mean by

$$
p_{\text {viol }} \cong \frac{N_{H}}{N}
$$

where $N_{H}$ is the number of successful simulations and $N$ the total number of simulations.

\section{Numerical results}

For the purposes of this study two 3D steel truss structures have been considered. For both test examples, two objective functions have been taken into account, the weight of the structure and the standard deviation of a characteristic node displacement representing the response of the structure, subject to constraints on stresses, element buckling and displacements imposed by the European design codes [9]. The design variables considered are the dimensions of the members of the structure, taken from the Circular Hollow Section (CHS) table of the Eurocode. The random variables related to the cross sections are the external diameter $D$ and the thickness $t$ of the circular hollow section. Apart from the cross-sectional dimensions of the structural members, the material properties (modulus of elasticity $E$ and yield stress $\sigma_{y}$ ) and the lateral loads have been considered as random variables.

\subsection{Three dimensional 39-bar truss}

The first test example considered is the 39-bar truss structure shown in Figure 3. The height of the structure is $16 \mathrm{~m}$, while its basis is an equilateral triangle of side $6.93 \mathrm{~m}$. The model consists of 15 nodes and 39 elements divided into 4 design variables. A vertical load $V=2 \mathrm{kN}$ is applied to all nodes, while a probabilistic horizontal load $F$ of mean value $8 \mathrm{kN}$ is applied to the top nodes at the $x$-direction. The type of probability density function, the mean value, and the variance of the random variables are shown in Table 1.

In order to examine the performance of the MCS combined with LHS a parametric study is performed that monitors the influence of the number of LHS simulations with respect to the accuracy in the calculation of the statistical quantities. For this reason, the probability of violation of the displacement constraint and the standard deviation of the characteristic node displacement are calculated with respect to the number of LHS simulations for the case of a randomly selected design vector. The results of this study can be seen in Figure 4. For this test example 3,000 LHS simulations have been considered enough in order to calculate with sufficient accuracy the statistical quantities under consideration. 


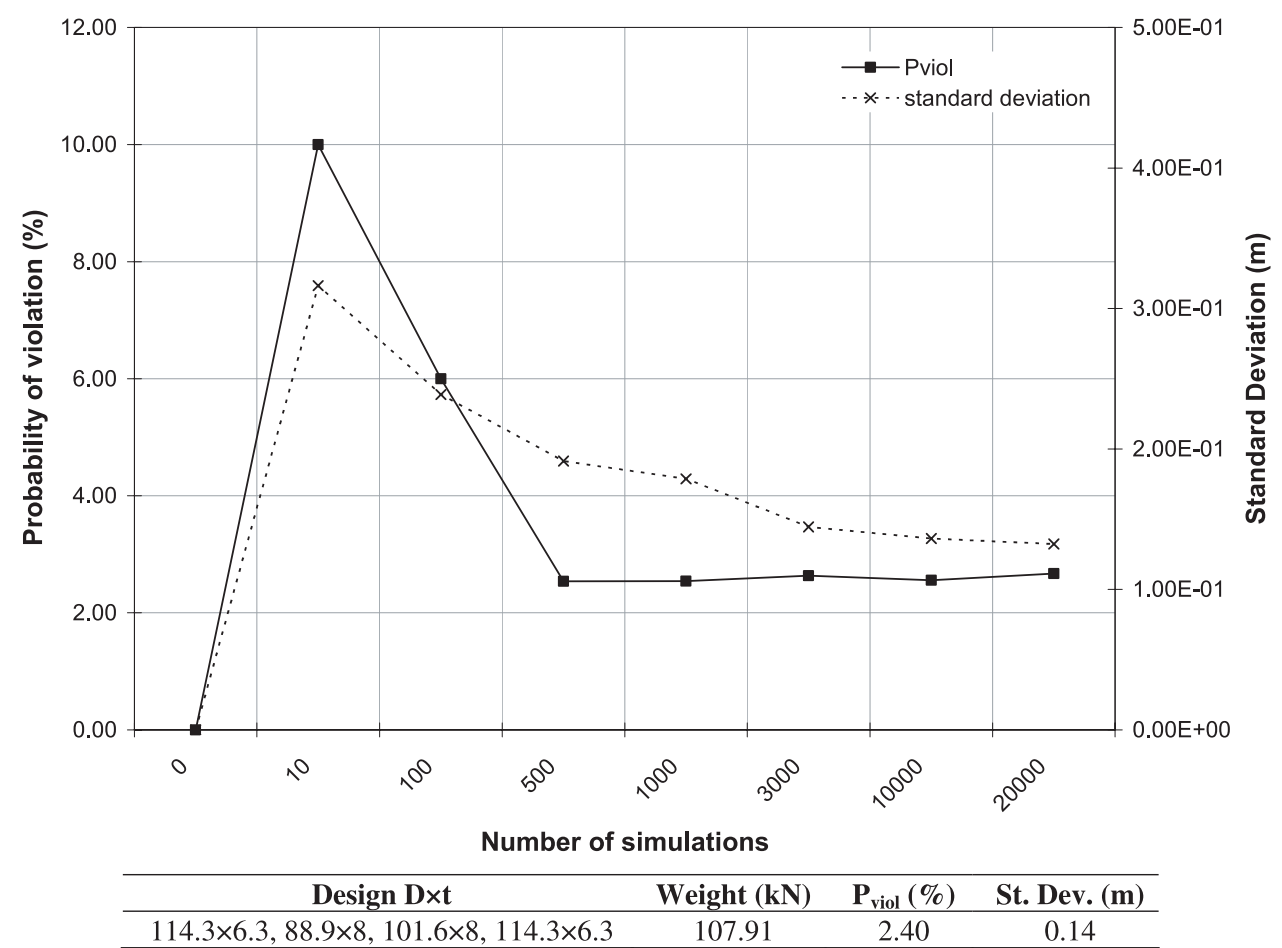

Fig. 4. 39-bar truss: Verification.

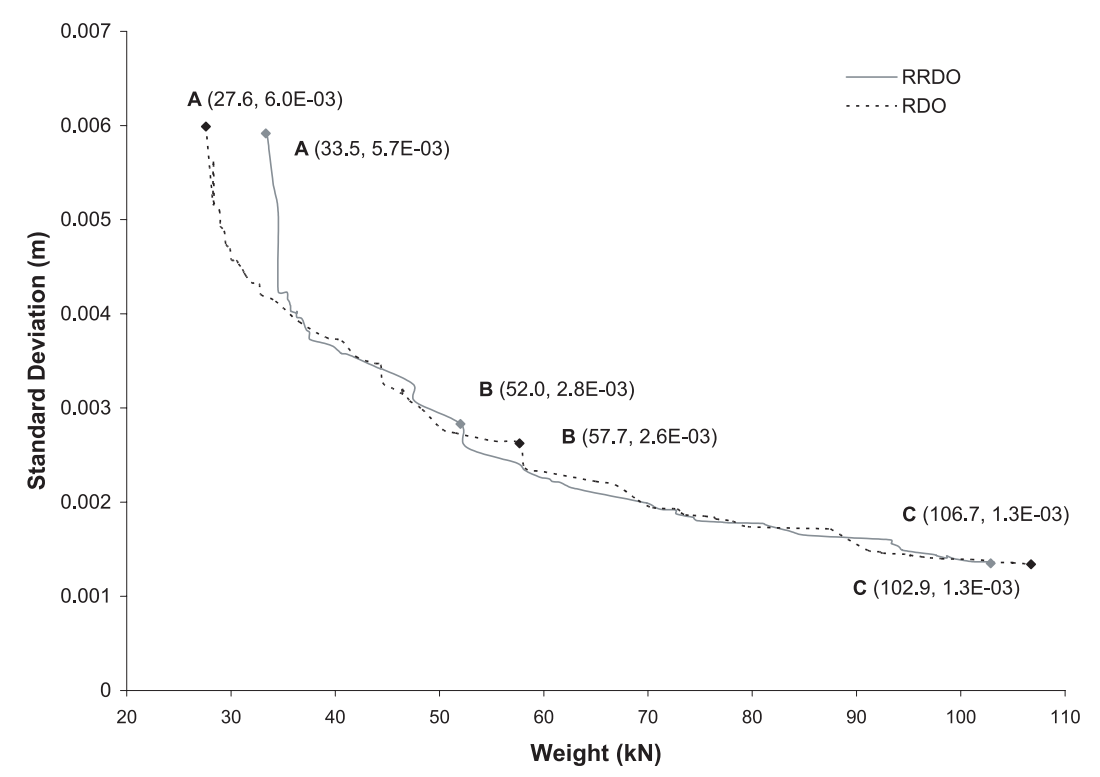

Fig. 5. 39-bar truss: Comparison of the Pareto front curves.

For the solution of the multi-objective optimization problem in question the non-dominant $C E A T m(\mu+$ $\lambda)_{\text {nrun,csteps }}$ optimization scheme was employed where $\mu=\lambda=5$, nrun $=10$ and csteps $=3$. These values were found in a previous study by the authors [16] to be appropriate for providing good quality Pareto front curves. Two different formulations of the RDO problem have been considered in this study: (i) RRDO with probabilistic constraints and (ii) the RDO formulation. In the case that probabilistic constraints have been taken into account, a probability of violation equal to $2 \%$ has been considered. The resultant Pareto front curves for the two RDO formulations are depicted in Figure 5, with the structural weight on the horizontal axis and the standard deviation of the characteristic node displacement on the vertical axis.

It can be seen from Figure 5 that the influence of considering the probabilistic constraints is significant when the weight of the structure is the dominant criterion (designs A). The two Pareto front curves almost coincide 
Table 2. 39-bar truss example: characteristic optimal solutions.

\begin{tabular}{ccccccc}
\hline & \multicolumn{3}{c}{ RDO } & \multicolumn{3}{c}{ RRDO } \\
\cline { 2 - 7 }$(\mathbf{D} \times \mathbf{t})^{*}$ & $\begin{array}{c}\mathbf{D B O}(\mathbf{A}) \\
(\mathbf{D} \times \mathbf{t})^{*}\end{array}$ & $\begin{array}{c}(\mathbf{B}) \\
(\mathbf{D} \times \mathbf{t})^{*}\end{array}$ & $\begin{array}{c}(\mathbf{C}) \\
(\mathbf{D} \times \mathbf{t})^{*}\end{array}$ & $\begin{array}{c}\mathbf{D B O}(\mathbf{A}) \\
(\mathbf{D} \times \mathbf{t})\end{array}$ & $\begin{array}{c}\mathbf{( B )} \\
(\mathbf{D} \times \mathbf{t})^{*}\end{array}$ & $\begin{array}{c}(\mathbf{C}) \\
(\mathbf{D} \times \mathbf{t})^{*}\end{array}$ \\
\hline $\mathrm{Sec}_{1}$ & $139.7 \times 4$ & $168.3 \times 6.3$ & $193.7 \times 12.5$ & $139.7 \times 5$ & $139.7 \times 10$ & $193.7 \times 12.5$ \\
$\mathrm{Sec}_{2}$ & $139.7 \times 5$ & $219.1 \times 7.1$ & $193.7 \times 12.5$ & $168.3 \times 4.5$ & $114.3 \times 10$ & $193.7 \times 12.5$ \\
$\mathrm{Sec}_{3}$ & $101 \times 6.3$ & $168.3 \times 8$ & $193.7 \times 12.5$ & $101.6 \times 10$ & $139.7 \times 8$ & $193.7 \times 12.5$ \\
$\mathrm{Sec}_{4}$ & $139.7 \times 4$ & $193.7 \times 6.3$ & $193.7 \times 12.5$ & $139.7 \times 4$ & $101.6 \times 10$ & $273 \times 7.1$ \\
Weight $(\mathrm{kN})$ & 27.6 & 57.7 & 106.7 & 33.5 & 52.0 & 102.9 \\
Variance $(\mathrm{m})$ & $6.04 \mathrm{E}-03$ & $2.62 \mathrm{E}-03$ & $1.34 \mathrm{E}-03$ & $5.71 \mathrm{E}-03$ & $2.83 \mathrm{E}-03$ & $1.35 \mathrm{E}-03$ \\
$\mathrm{P}_{\text {viol }}(\%)$ & $4.2 \mathrm{E}-00$ & $3.9 \mathrm{E}-01$ & $9.0 \mathrm{E}-03$ & $1.9 \mathrm{E}-00$ & $5.1 \mathrm{E}-01$ & $9.0 \mathrm{E}-03$ \\
\hline
\end{tabular}

* Taken from the Circular Hollow Section (CHS) table of the Eurocode.

when the importance of the second criterion (standard deviation of the response) increases. In Table 2 three designs are compared which have been selected from the two Pareto front curves. Designs $B_{R R D O}$ versus $B_{R D O}$ and $C_{R R D O}$ versus $C_{R D O}$ are similar, with respect to both weight and standard deviation of the response, leading to similar probabilities of violation. On the other hand designs $A_{R R D O}$ and $A_{R D O}$ differ by $17.5 \%$ with respect to the weight and by $6.0 \%$ with respect to the standard deviation of the characteristic node displacement. Moreover the probability of violation of the constraints, in the case of the $A_{R D O}$ design, is equal to $4.2 \%$ while it becomes equal to $1.9 \%$ for the $A_{R R D O}$ design case.

\subsection{Three dimensional truss tower}

The second test example considered is the $3 \mathrm{D}$ truss tower shown in Figure 6. The height of the truss tower is $128 \mathrm{~m}$, while its basis is a rectangle of side $17.07 \mathrm{~m}$. The model consists of 324 nodes and 1254 elements divided into 12 groups that play the role of the design variables. The applied loading consists of: (i) self weight (dead load), (ii) live loads and (iii) wind actions according to the Eurocode [20]. The type of probability density function, the mean value, and the variance of the random variables are shown in Table 3.

A similar to the previous test example parametric study, is also performed here, in order to examine the applicability of the MCS with LHS. The probability of violation of the displacement constraint and the standard deviation of the characteristic node displacement are calculated with respect to the number of LHS simulations for the case of a randomly selected design vector. The results of this test example are depicted in Figure 7. For this test example 3,000 LHS simulations have also been considered enough in order to calculate with sufficient accuracy the statistical quantities.

For the solution of the multi-objective optimization problem in question the non-dominant $C E A T m(\mu+$ $\lambda)_{\text {nrun,csteps }}$ optimization scheme was employed where $\mu=\lambda=5$, nrun $=10$ and csteps $=3$. The resultant Pareto front curves for the two RDO formulations are depicted in Figure 8. As it can be seen from Figure 8

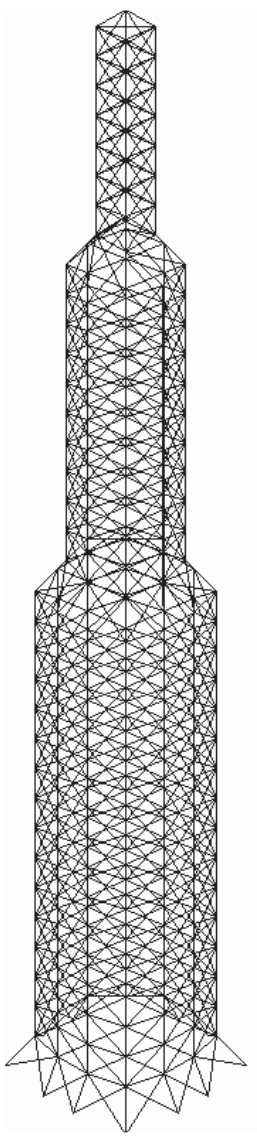

(a)

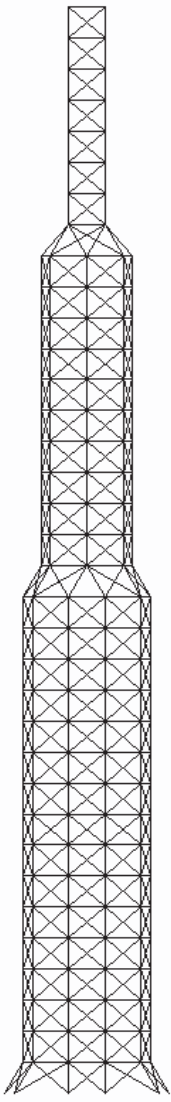

(b)

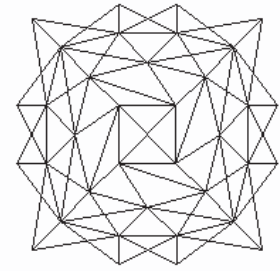

(c)
Fig. 6. 3D truss tower: (a) 3D view, (b) side view, (c) top view.

the trend on the influence of considering the probabilistic constraints is similar to the one of the first test example, i.e. it is significant near the area where the weight of the structure is the dominant criterion. When the importance of the standard deviation of the response increases, the two Pareto front curves almost coincide. In Table 4 three designs are compared which have been selected from the two Pareto front curves. Designs $\mathrm{B}_{R R D O}$ versus $\mathrm{B}_{R D O}$ and $\mathrm{C}_{R R D O}$ versus $\mathrm{C}_{R D O}$ are similar with respect to both weight and standard deviation of the response while they 
Table 3. 3D truss tower example: characteristics of the random variables.

\begin{tabular}{ccccc}
\hline \multicolumn{2}{c}{ Random variable } & $\begin{array}{c}\text { Probability } \\
\text { Density } \\
\text { Function }\end{array}$ & $\begin{array}{c}\text { Mean value } \\
\mu\end{array}$ & CoV \\
\hline $\mathbf{E}\left(\mathbf{k N} / \mathbf{m}^{2}\right)$ & Young's Modulus & Normal & $2.10 \mathrm{E}+08$ & $7.14 \%$ \\
$\sigma_{y}\left(\mathbf{k N} / \mathbf{m}^{2}\right)$ & Allowable stress & Normal & $3.55 \mathrm{E}+05$ & $10.00 \%$ \\
$\mathbf{F ~}\left(\mathbf{k N} / \mathbf{m}^{2}\right)$ & Wind loading & Normal & $\mathrm{F}_{\mu}$ & $40.00 \%$ \\
$\mathbf{D}$ & CHS Diameter & Normal & $\mathrm{d}_{i} *$ & $2 \%$ \\
$\mathbf{t}$ & CHS Thickness & Normal & $\mathrm{t}_{i} *$ & $2 \%$ \\
\hline
\end{tabular}

* Taken from the Circular Hollow Section (CHS) table of the Eurocode, for every design.

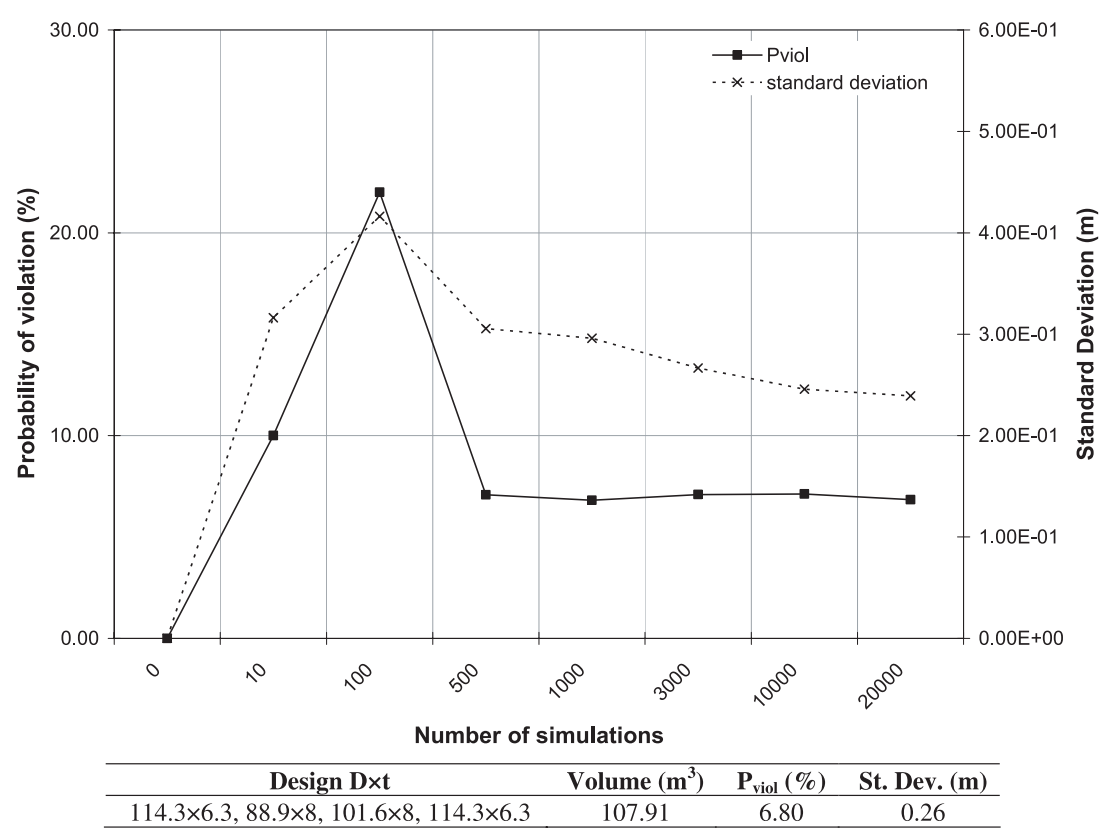

Fig. 7. 3D truss tower: verification.

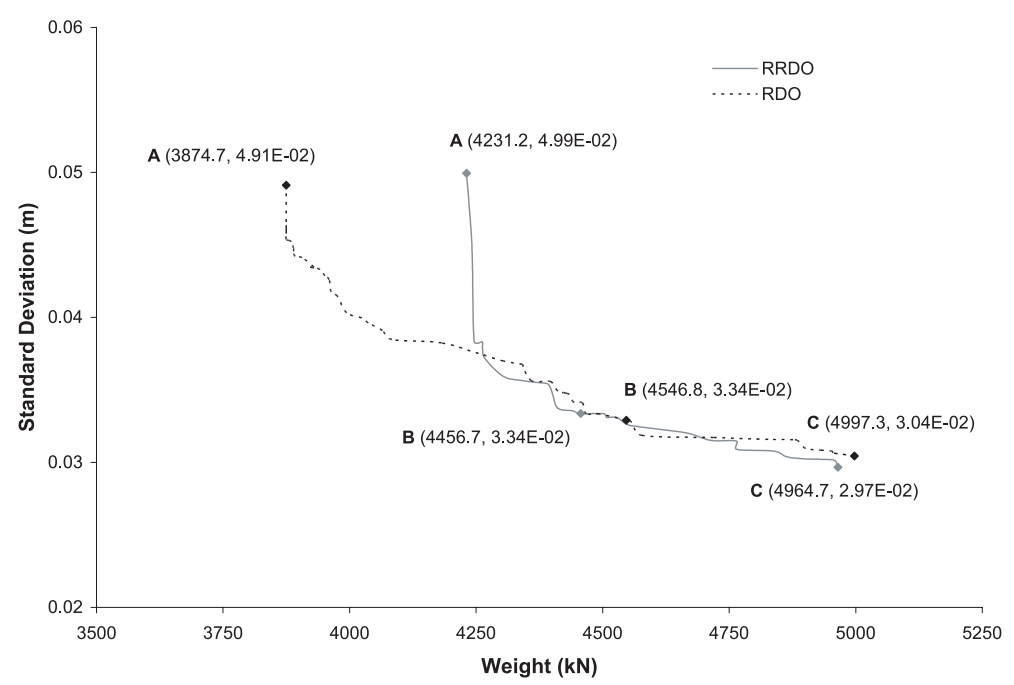

Fig. 8. 3D truss tower: comparison of the Pareto front curves. 
Table 4. 3D truss tower example: characteristic optimal solutions.

\begin{tabular}{|c|c|c|c|c|c|c|}
\hline \multirow[b]{2}{*}{$(\mathrm{D} \times \mathrm{t})^{*}$} & \multicolumn{3}{|c|}{ RDO } & \multicolumn{3}{|c|}{ RRDO } \\
\hline & $\begin{array}{c}\text { DBO (A) } \\
(\mathrm{D} \times \mathrm{t})^{*}\end{array}$ & $\begin{array}{c}(\mathrm{B}) \\
(\mathrm{D} \times \mathrm{t})^{*}\end{array}$ & $\begin{array}{c}(\mathrm{C}) \\
(\mathrm{D} \times \mathbf{t})^{*}\end{array}$ & $\begin{array}{c}\text { DBO (A) } \\
(\mathrm{D} \times \mathrm{t})^{*}\end{array}$ & $\begin{array}{c}(\mathrm{B}) \\
(\mathrm{D} \times \mathrm{t})^{*}\end{array}$ & $\begin{array}{c}(\mathbf{C}) \\
(\mathbf{D} \times \mathbf{t})^{*}\end{array}$ \\
\hline $\mathrm{Sec}_{1}$ & $355.6 \times 8.0$ & $219.1 \times 20.0$ & $406.4 \times 10.0$ & $323.9 \times 8.0$ & $323.9 \times 8.0$ & $323.9 \times 12.5$ \\
\hline $\mathrm{Sec}_{3}$ & $355.6 \times 10.0$ & $355.6 \times 10.0$ & $406.4 \times 8.8$ & $355.6 \times 10.0$ & $355.6 \times 10.0$ & $323.9 \times 12.5$ \\
\hline $\mathrm{Sec}_{4}$ & $323.9 \times 10.0$ & $244.5 \times 16.0$ & $323.9 \times 12.5$ & $355.6 \times 10.0$ & $219.1 \times 20.0$ & $406.4 \times 8.8$ \\
\hline $\mathrm{Sec}_{5}$ & $406.4 \times 8.8$ & $406.4 \times 8.8$ & $406.4 \times 8.8$ & $406.4 \times 8.8$ & $323.9 \times 12.5$ & $406.4 \times 8.8$ \\
\hline $\mathrm{Sec}_{6}$ & $355.6 \times 8.0$ & $355.6 \times 8.0$ & $355.6 \times 10.0$ & $355.6 \times 8.0$ & $355.6 \times 8.0$ & $273.0 \times 12.5$ \\
\hline $\mathrm{Sec}_{7}$ & $323.9 \times 8.0$ & $273.0 \times 8.0$ & $323.9 \times 10.0$ & $323.9 \times 8.0$ & $323.9 \times 8.0$ & $219.1 \times 16.0$ \\
\hline $\mathrm{Sec}_{8}$ & $323.9 \times 10.0$ & $323.9 \times 12.5$ & $244.5 \times 20.0$ & $355.6 \times 10.0$ & $355.6 \times 10.0$ & $273.0 \times 16.0$ \\
\hline $\mathrm{Sec}_{9}$ & $323.9 \times 8.0$ & $323.9 \times 8.0$ & $355.6 \times 8.0$ & $323.9 \times 8.0$ & $323.9 \times 8.0$ & $323.9 \times 8.0$ \\
\hline $\mathrm{Sec}_{10}$ & $219.1 \times 7.1$ & $323.9 \times 8.0$ & $273.0 \times 12.5$ & $355.6 \times 8.0$ & $355.6 \times 8.0$ & $219.1 \times 20.0$ \\
\hline $\operatorname{Sec}_{11}$ & $323.9 \times 10.0$ & $244.5 \times 20.0$ & $244.5 \times 20.0$ & $406.4 \times 8.8$ & $244.5 \times 20.0$ & $244.5 \times 20.0$ \\
\hline $\mathrm{Sec}_{12}$ & $323.9 \times 8.0$ & $323.9 \times 12.5$ & $273.0 \times 16.0$ & $323.9 \times 10.0$ & $273.0 \times 12.5$ & $273.0 \times 16.0$ \\
\hline Weight $(\mathrm{kN})$ & 3874.7 & 4546.8 & 4997.3 & 4231.2 & 4456.7 & 4964.7 \\
\hline Variance $(\mathrm{m})$ & 4.91E-02 & $3.34 \mathrm{E}-02$ & $3.04 \mathrm{E}-02$ & 4.99E-02 & 3.34E-02 & $2.97 \mathrm{E}-02$ \\
\hline $\mathrm{P}_{\text {viol }}(\%)$ & $3.8 \mathrm{E}-00$ & $2.8 \mathrm{E}-01$ & $8.5 \mathrm{E}-03$ & $1.8 \mathrm{E}-00$ & $2.9 \mathrm{E}-01$ & $8.0 \mathrm{E}-03$ \\
\hline
\end{tabular}

* Taken from the Circular Hollow Section (CHS) table of the Eurocode.

have similar probabilities of violation. On the other hand designs $\mathrm{A}_{R R D O}$ and $\mathrm{A}_{R D O}$ differ by $10.0 \%$ with respect to the weight and by $2.0 \%$ with respect to the standard deviation of the characteristic node displacement. Moreover the probability of violation of the constraints, in the case of the $\mathrm{A}_{R D O}$ design, is equal to $3.8 \%$ while it becomes equal to $1.8 \%$ for the $\mathrm{A}_{R R D O}$ design.

\section{Concluding remarks}

In most cases optimum design of structures is based on deterministic parameters and is focused on the satisfaction of the deterministically defined code provisions. The deterministic optimum is not always a "safe" design, since many random factors might affect the design, the manufacturing and the performance of a structure during its lifetime. In order to find the "real" optimum the designer has to take into account all necessary random parameters. For this purpose two separate formulations have been proposed in the past: the reliability based optimization and the robust design optimization. In the present work the reliability based robust design optimization formulation is examined where probabilistic constraints are incorporated into the robust design optimization formulation.

The Pareto front curves obtained for the RRDO and RDO formulations appear to be quite distinct when the weight objective function is predominant. In particular for the same standard deviation the weight of the RRDO optimum solution is larger compared to the optimum solution achieved through the RDO formulation. The probability of violation of the constraints of the RRDO solution is up to $60 \%$ lower than the one of violation of the RDO solution. Furthermore it was observed that considering the standard deviation of the response as an additional objective function forces the solution of the RDO formulation to coincide with to of the RRDO formulation.

Acknowledgements. This work has been funded by the project PENED 2003. The project is part of the Operational Programme "Competitiveness" (measure 8.3) of the 3rd Community Support Programme and is co-funded, $75 \%$ of public expenditure through EC - European Social Fund, 25\% of public expenditure through the Greek Ministry of Development General Secretariat of Research and Technology and through private sector.

\section{References}

1. G.I. Schueller, Computational stochastic mechanics - recent advances, Comp. \& Struct. 79, 2225-2234 (2001)

2. K.-H. Lee, G.-J. Park, Robust optimization considering tolerances of design variables. Comput. Struct. 79, 77-86 (2001)

3. A. Messac, A. Ismail-Yahaya, Multiobjective robust design using physical programming. Struct. Multidiscip. O. 23, 357-371 (2002)

4. Hans-Georg Beyer, Bernhard Sendhoff, Robust optimization - A comprehensive survey. Comput. Method. Appl. M. 196, 3190-3218 (2007)

5. M. Papadrakakis, N.D. Lagaros, Reliability-based structural optimization using neural networks and Monte Carlo simulation. Comput. Method. Appl. M. 191, 3491-3507 (2002)

6. X. Qu, R.T. Haftka, S. Venkataraman, Deterministic and Reliability-Based Optimization of Composite Laminates for Cryogenic Environments. AIAA Journal 41, 2029$2036(2003)$

7. M. Allen, K. Maute, Reliability-based shape optimization of structures undergoing fluid-structure interaction phenomena. Comput. Method. Appl. M. 194 (30-33), 34723495 (2005) 
8. J.S. Kong, D.M. Frangopol, Life-cycle reliabilitybased maintenance cost optimization of deteriorating structures with emphasis on bridges. Journal of Structural Engineering - ASCE 129, 818-828 (2003)

9. Eurocode 3, Design of steel structures, Part1.1: General rules for buildings, CEN, ENV 1993-1-1/1992

10. V. Pareto, Cours d'économique politique, Vol. 1\&2, Rouge, Lausanne (1896)

11. R.T. Marler, J.S. Arora, Survey of Multi-objective Optimization Methods for Engineering. Structural and Multidisciplinary Optimization 26, 369-395 (2004)

12. C.A. Coello Coello, An Updated Survey of GABased Multi-objective Optimization Techniques. ACM Computing Surveys 32, 109-143 (2000)

13. J. Nocedal, S.J. Wright, Numerical Optimization, Springer, New York (1999)

14. N.D. Lagaros, M. Papadrakakis, G. Kokossalakis, Structural optimization using evolutionary algorithms. Comput. Struct. 80, 571-587 (2002)

15. M. Papadrakakis, Y. Tsompanakis, N.D. Lagaros, Structural shape optimization using Evolution Strategies. Eng. Optimiz. 31, 515-540 (1999)
16. N.D. Lagaros, V. Plevris, M. Papadrakakis, Multiobjective design optimization using cascade evolutionary computations. Comput. Method. Appl. M. 194, 3496-3515 (2005)

17. S.N. Patnaik, R.M. Coroneos, J.D. Guptill, D.A. Hopkins, Comparative evaluation of different optimization algorithms for structural design applications. Int. J. Numer. Meth. Eng. 39, 1761-1774 (1996)

18. D.C. Charmpis, N.D. Lagaros, M. Papadrakakis, Multidatabase exploration of large design spaces in the framework of cascade evolutionary structural sizing optimization. Comput. Method. Appl. M. 194 (30-33) 3315-3330 (2005)

19. M.D. McKay, R.J. Beckman, W.J. Conover, A comparison of three methods for selecting values of input variables in the analysis of output from a computer code, Technometrics 21, 239-245 (1979)

20. Eurocode 1 : Basis of design and actions on structures - Part 2-4: Actions on Structures - Wind actions, CEN, ENV 1991-2-4, May 1995 